\title{
Nomograms for Predicting the Overall and Cancer-Specific Survival of Patients with High-Grade Glioma: A Surveillance, Epidemiology, and End Results Study
} \author{
Yingjie JIANG $^{3}$, Jun WANG ${ }^{2,4}$ \\ ${ }^{1}$ Basic Medical College, Southern Medical University, Guangdong, China \\ ${ }^{2}$ First Clinical Medical School, Nanfang Hospital, Southern Medical University, Guangzhou, China \\ ${ }^{3}$ Basic Medical College, Navy Medical University, No.168 Changhai Road, Shanghai, China \\ ${ }^{4}$ Department of Neurosurgery, Nanfang Hospital, Southern Medical University, Guangzhou, China \\ ${ }^{*}$ These authors contributed equally.
}

Yuhan XIA ${ }^{1^{*}}$, Weixin $\mathrm{LIAO}^{2 *}$, Shaozhuo HUANG ${ }^{2}$, Zhicheng LIU², Xiaowen HUANG ${ }^{2}$, Chen YANG ${ }^{2}$, Chao YE

Corresponding author: Jun WANG smuwangjun@163.com

\section{ABSTRACT}

AIM: To predict the overall survival (OS) and the cancer-specific survival (CSS) of patients with high-grade glioma (HGG) using nomograms and the surveillance, epidemiology, and end results (SEER) database (2000-2013).

MATERIAL and METHODS: A total of 3706 patients with high-grade glioma were identified by the SEER database (2000-2013). Based on the relevant information of these patients, we divided the primary cohort into a training cohort $(n=3336)$ and a validation cohort $(n=370)$. The nomograms were constructed by the training cohort and corroborated by the validation cohort.

RESULTS: According to the multivariate analysis of the training cohort, the nomograms of OS and CSS indicated that patient age at diagnosis, laterality, radiation, and the extent of resection are significantly correlated with the survival rate. The c-indexes of the nomograms of OS and CSS of the training cohort are 0.682 [95\% confidence interval (Cl): $0.671-0.693$ ] and 0.678 (95\% Cl: 0.666 0.690), respectively. The calibration curve plots of 1- and 3-year OS and CSS showed that the nomogram predictions are consistent with the observed outcomes for both the training and validation cohorts.

CONCLUSION: Based on the data obtained, we established a scoring model to predict the OS and the CSS of patients with HGG. All calibration curves showed high consistency between the predicted and actual survival.

KEYWORDS: Brain tumors, Glioma, Nomogram, Overall survival, Cancer-specific survival

\section{INTRODUCTION}

$\mathrm{H}$ igh-grade glioma (HGG) is the most common primary brain tumor in the central nervous system. It can be classified into four different grades according to the World Health Organization (WHO) classification system (15). HGG comprises both grade III (anaplastic astrocytoma or anaplastic oligodendroglioma) and grade IV (glioblastoma)
(17). Despite the recent advances in surgical techniques, radiation, and adjunctive therapies, the prognosis of patients with HGG is still poor, with a median survival of 12 to 14 months after diagnosis (17). Since to date there are no specific studies that have investigated the prognosis of HGG, we established nomograms to predict the overall survival (OS) and cancerspecific survival (CSS) with the aim to develop an applicable prognostic evaluation system for HGG.
Yuhan XIA $\quad$ (D) : 0000-0002-1617-4246

Weixin LIAO (1) : 0000-0001-5272-1510

Shaozhuo HUANG (1) : 0000-0002-9272-2497
Zhicheng LIU (D) : 0000-0002-5950-409X

Xiaowen HUANG : 0000-0002-2671-1682

Chen YANG : 0000-0002-1237-2632
Chao YE

(D) : 0000-0002-4157-7557

Yingjie JIANG (1D) : 0000-0001-6742-2038

Jun WANG (10): 0000-0003-3509-0790 
The surveillance, epidemiology and end results (SEER) database from the U.S. National Cancer Institute contains data on patient demographics, primary tumor sites, treatment information, and survival rate. The database includes data from $28 \%$ of the population. Notably, it avoids potentially limiting biases that result when evaluating patients from a single institution by allowing the usage of patient data from multiple institutions (19).

The nomogram is a graphical representation of a complex mathematical formula. When a carefully constructed nomogram is appropriately interpreted and applied, its value to clinical practice can never be overstated (2). Gorlia et al. used nomograms to predict the survival of patients with newly diagnosed glioblastoma (7). Zhang et al. used nomograms to predict OS and CSS of patients with classical Hodgkin lymphoma (30). Through the integration of biological and clinical variables, medical nomograms can determine a statistical prognostic model that could generate a probability of a clinical event, such as cancer recurrence or death, for any particular individual. Therefore, the nomogram plays a critical role in predicting the clinical outcomes of the tumor $(14,24,30)$. These new prognostic models can help improve the predictions of survival outcomes, resulting in reasonable treatment protocols and recommendations.

Therefore, the aim of this study was to use the SEER database to develop comprehensive and practical nomograms that can generate precise estimations of OS and CSS for patients with HGG. To our current knowledge, this study is the latest and the most comprehensive clinical guiding principle for the prognosis of adult HGG.

\section{MATERIAL and METHODS}

The data of patients with HGG diagnosed from 2000 to 2013 were retrieved from the SEER registry database. The SEER database collects demographic, diagnostic, and treatment information of cancer patients, such as primary sites, morphology, stage, surgery, radiotherapy, and patients' vital status in 18 registries within the United States. By using SEER ${ }^{\star}$ Stat software (Version 8.3.2), we retrieved 40,063 cases in total. Only patients who met the following inclusion criteria were selected: 1) diagnosed with glioblastoma multiforme (GBM) as his or her first and only malignancy; 2) the histological type was limited to glioblastoma (9440-9442) according to the ICD-O-3 histological codes; 3) the ICD-O-3 site codes were limited to C71.0 (Cerebrum), C71.1 (Frontal lobe), C71.2 (Temporal lobe), C71.3 (Parietal lobe), C71.4 (Occipital lobe), C71.6 (Cerebellum, NOS), and C71.7 (Brain stem) according to the SEER classification; 4) included definite information on race, radiotherapy, and tumor size.

At the same time, patients were excluded in any of the following cases: 1) diagnosis was obtained through death certificate or autopsy only; 2) age at diagnosis was less than 20 years; 3) diagnosed with grade I, II, or unknown glioblastoma; 4) patient received no surgery, local tumor destruction, or biopsy, or the surgery status was unknown (Figure 1).
A total of 3706 patients with HGG were randomized into two groups (training cohort $n=3336$ and validation cohort $n$ $=370$ ) to develop and validate nomograms. Nine important clinicopathological factors of patients with HGG were used to conduct the univariate and multivariate analysis: age at diagnosis, gender, race, marital status, tumor location, laterality, size, radiation, and surgery. We categorized age into two groups: less than 60 years, and 60 years or more. Race was categorized as white, black, and others. Marital status was classified as unmarried, married, and unknown. We divided patients into two groups by radiation status: radiation after surgery and others, as radiation after surgery was the standard treatment for patients with HGG (21). A SEER variable "RX Summ-Surg Prim Site" was used to determine the type of surgery and the extent of resection [gross total resection (GTR) and no GTR].

One of our primary endpoints of interest was the median OS time, measured as the time from diagnosis to death from all possible causes. In the analysis of OS, patients who were alive at the time of the last follow-up were treated as censored observations. The other primary endpoint of interest was the median CSS time, which was defined as the time from diagnosis to death attributed to glioblastoma. In the analysis of CSS, patients who died from other causes or were alive at the last follow-up were treated as censored observations.

To determine survival-related factors, we calculated and divided OS and CSS into 1-year survival group, 3-year survival group, and 5-year survival group; we constructed 95\% confidence intervals (Cls) for each subgroup. The entire study used the Kaplan-Meier method for sampling. The nomograms were constructed based on the Cox proportional hazards regression model to predict 1-, 3-, and 5-year OS and CSS, whereas the factors had significant correlations with the survival rate in univariate or multivariate analyses $(p<0.05)$.

The nomograms were constructed using 1000 bootstrap samples for both internal and external validations in the training and validation cohort. The Harrell concordance index (C-index) was used to assess the model's predictive performance, which was similar to the area under the curve (AUC) but was more suitable for censored data (8). The $\mathrm{C}$-index ranges from 0.5 to 1.0 , and a larger $\mathrm{C}$-index indicates more accurate prognostic predictions (20). In addition, we also used calibration curves to assess the model's predictive performance. And, in the perfectly calibrated model, predictions should fall on the diagonal $45^{\circ}$ line of the calibration plot.

\section{Statistical Analysis}

All analyses were performed using the SPSS 23.0 statistical software program (SPSS Inc., Chicago, IL, USA) and R 3.2.2 software (Institute for Statistics and Mathematics, Vienna, Austria) (http://www.r-project.org/). The KapanMeier method was used to estimate the survival time; the log-rank test was used for survival comparisons; and the multivariable Cox proportional hazard regression was used to identify associations with outcome variables. The nomogram for prognostic factors associated with OS and CSS was established using $\mathrm{R}$ software. The predictive accuracy 


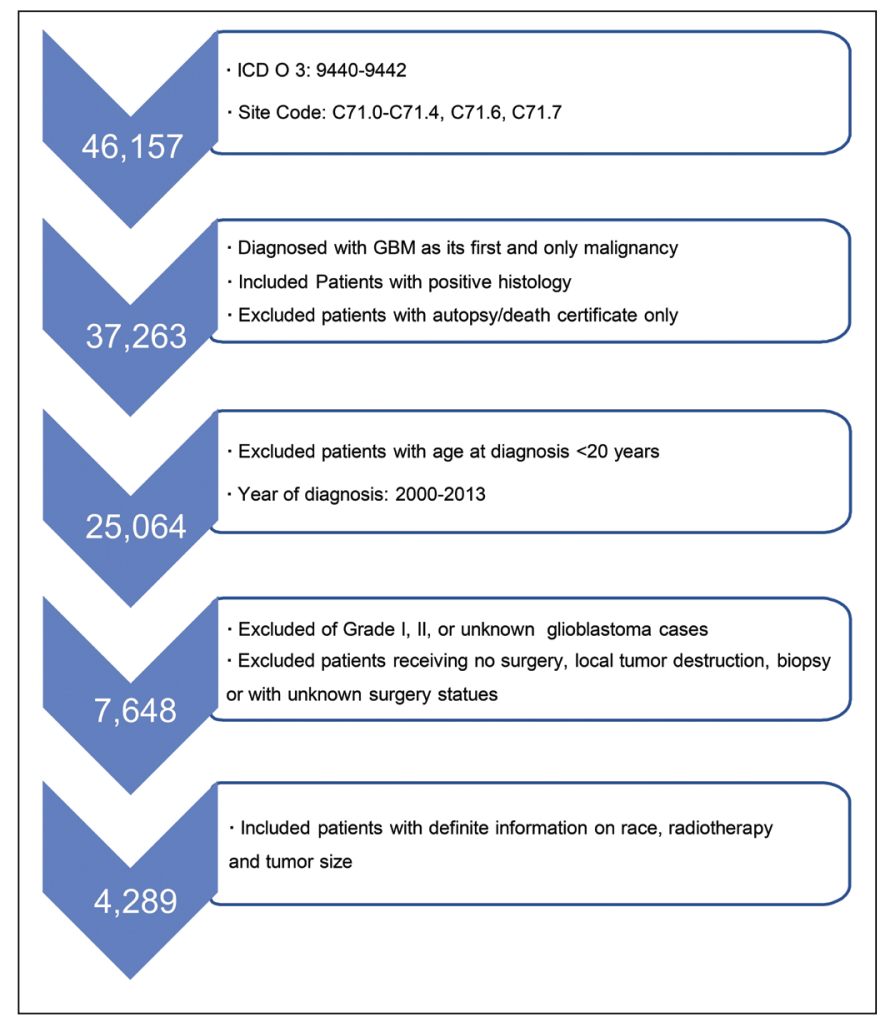

Figure 1: Data selection flowchart.

was evaluated based on the C-index using the rcorrp.cens package in Hmisc (11). A larger C-index was associated with a more accurate prediction (10). p values were two-sided, and $a=0.05$ was chosen as the statistical significance level.

\section{RESULTS}

\section{Patient baseline characteristics}

We randomly divided 3360 of the total of 3706 patients into the training cohort; and, the remainder of the patients were put into the validation cohort. The summary of the characteristics of the patients in the two cohorts is listed in Table I. There were no substantial differences between the two cohorts. The median of OS is 14.3 months (range, $1-118 \mathrm{mo}$ ), and the $1-$, 3-, and 5-year OS rates are $45.2 \%, 10.9 \%$, and $6.2 \%$, respectively. The 1-, 3-, and 5-year CSS rates are $47.6 \%$, $12.4 \%$, and $7.1 \%$, respectively (Table II).

\section{Independent prognostic factors in the training cohort}

Both the univariate and multivariate models were constructed based on the training cohort to determine significant predictors of CSS and OS. Age at diagnosis, marital status, laterality, radiation, and the extent of resection were found to be significantly correlated with CSS and OS in the univariate model using the Kaplan-Meier method (Figure 2A-E and 3A-E, respectively) and were further compared using the log-rank test $(p<0.05)$. Every predictor's effect on CSS and OS was assessed using the Cox proportional hazards regression. Age at diagnosis, laterality, radiation, and the extent of resection
Table I: Characteristics of the Training and Validation Cohorts

\begin{tabular}{|c|c|c|c|c|}
\hline \multirow[b]{2}{*}{ Characteristic } & \multicolumn{2}{|c|}{ Training Cohort } & \multicolumn{2}{|c|}{ Validation Cohort } \\
\hline & $\begin{array}{c}\text { No. of } \\
\text { Patients }\end{array}$ & $\%$ & $\begin{array}{c}\text { No. of } \\
\text { Patients }\end{array}$ & $\%$ \\
\hline \multicolumn{5}{|c|}{ Age at diagnosis } \\
\hline$<60$ years & 1490 & 44.7 & 163 & 44.1 \\
\hline$\geq 60$ years & 1846 & 55.3 & 207 & 55.9 \\
\hline \multicolumn{5}{|l|}{ Gender } \\
\hline Male & 1988 & 59.6 & 223 & 60.3 \\
\hline Female & 1348 & 40.4 & 147 & 39.7 \\
\hline \multicolumn{5}{|l|}{ Race } \\
\hline White & 3031 & 90.9 & 339 & 91.6 \\
\hline Black & 171 & 5.1 & 19 & 5.1 \\
\hline Others & 134 & 4.0 & 12 & 3.3 \\
\hline
\end{tabular}

\section{Marital status}

\begin{tabular}{lrrrr}
\hline Unmarried & 1069 & 32.1 & 122 & 33.0 \\
\hline Married & 2179 & 65.3 & 233 & 63.0 \\
\hline Unknown & 88 & 2.6 & 15 & 4.0 \\
\hline
\end{tabular}

\section{Location}

\begin{tabular}{lrrrr}
\hline Frontal lobe & 1203 & 36.1 & 126 & 34.0 \\
\hline Temporal lobe & 1145 & 34.3 & 132 & 35.7 \\
\hline Parietal lobe & 695 & 20.8 & 78 & 21.1 \\
\hline Occipital lobe & 185 & 5.6 & 25 & 6.8 \\
\hline Others & 108 & 3.2 & 9 & 2.4 \\
\hline
\end{tabular}

\section{Laterality}

\begin{tabular}{lrrrr}
\hline Right & 1712 & 51.3 & 179 & 48.4 \\
\hline Left & 1539 & 46.1 & 185 & 50.0 \\
\hline Bilateral/NOS & 85 & 2.6 & 6 & 1.6 \\
\hline
\end{tabular}

Size

\begin{tabular}{lrrrr}
\hline $0-3 \mathrm{~cm}$ & 699 & 21.0 & 85 & 23.0 \\
\hline $3.1-6 \mathrm{~cm}$ & 2091 & 62.7 & 228 & 61.6 \\
\hline$>6 \mathrm{~cm}$ & 546 & 16.3 & 57 & 15.4 \\
\hline
\end{tabular}

\section{Radiation}

\begin{tabular}{ccccc}
\hline $\begin{array}{l}\text { Radiation after } \\
\text { surgery }\end{array}$ & 2594 & 77.8 & 294 & 79.5 \\
\hline Others & 742 & 22.2 & 76 & 20.5 \\
\hline Extent of resection & & & & \\
\hline GTR & 1486 & 44.5 & 151 & 40.8 \\
\hline No GTR & 1850 & 55.5 & 219 & 59.2 \\
\hline
\end{tabular}

NOS: Not otherwise specified, GTR: Gross total resection. 
(A)

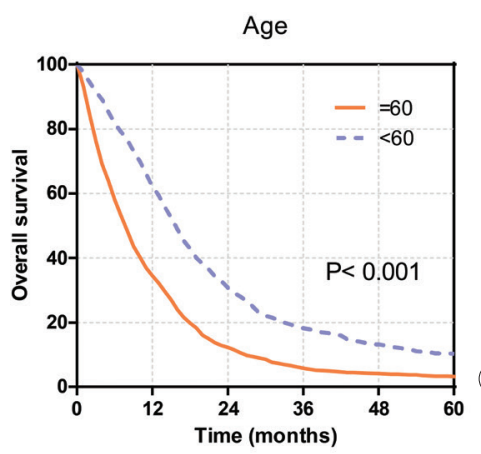

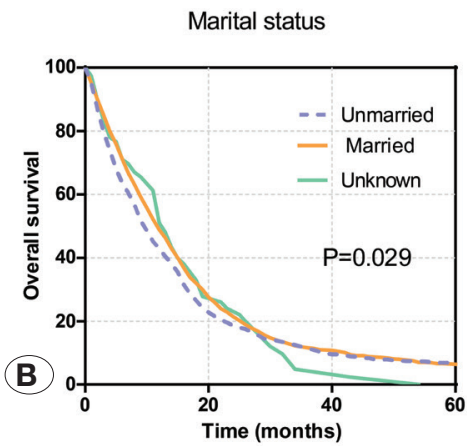

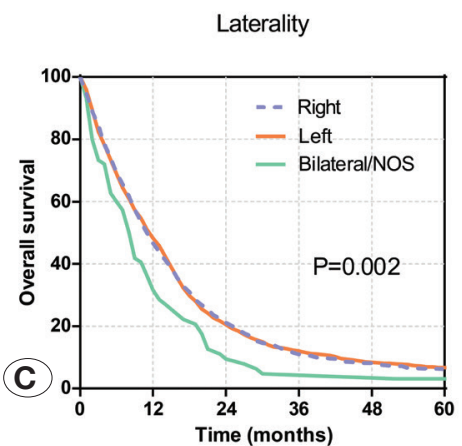

Radiation
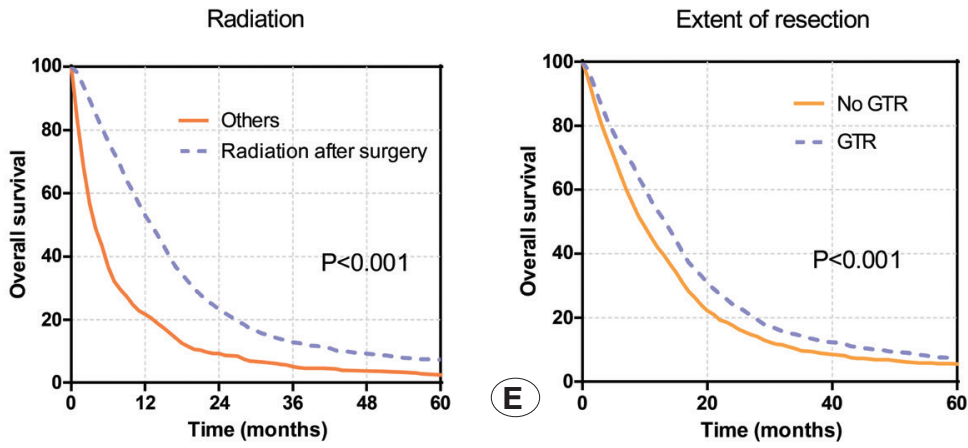

Figure 2: Kaplan-Meier survival curves for overall survival in the training cohort, as stratified by age, laterality, radiation, and extent of resection (A-E).

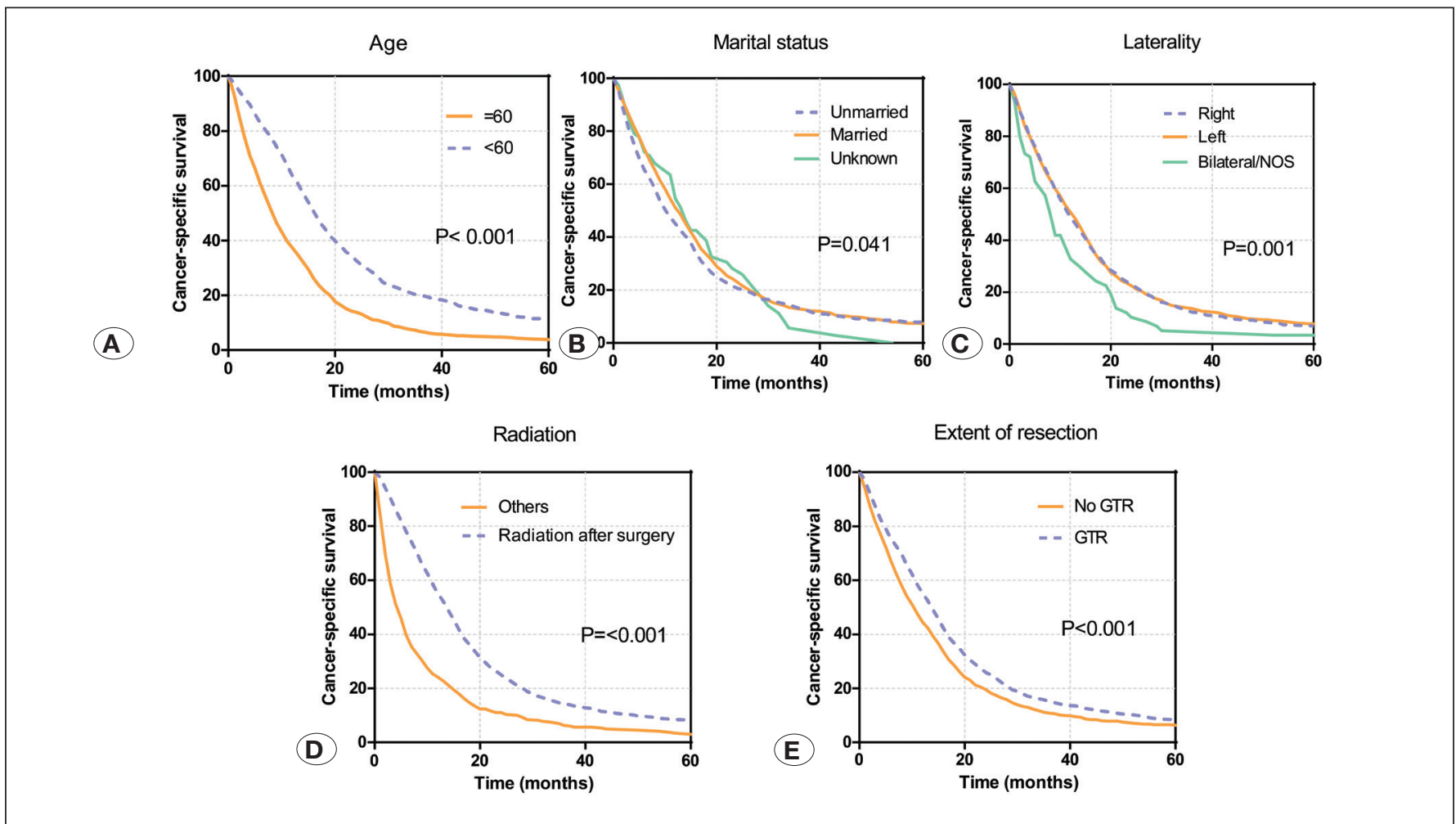

Figure 3: Kaplan-Meier survival curves for cancer-specific survival in the training cohort, as stratified by age, laterality, radiation, and extent of resection (A-E). 
Xia Y. et al: Predicting Survival Rates for High-Grade Glioma

Table II: 1-, 3- and 5-Year Overall and Cancer-Specific Survival in the Training Cohort

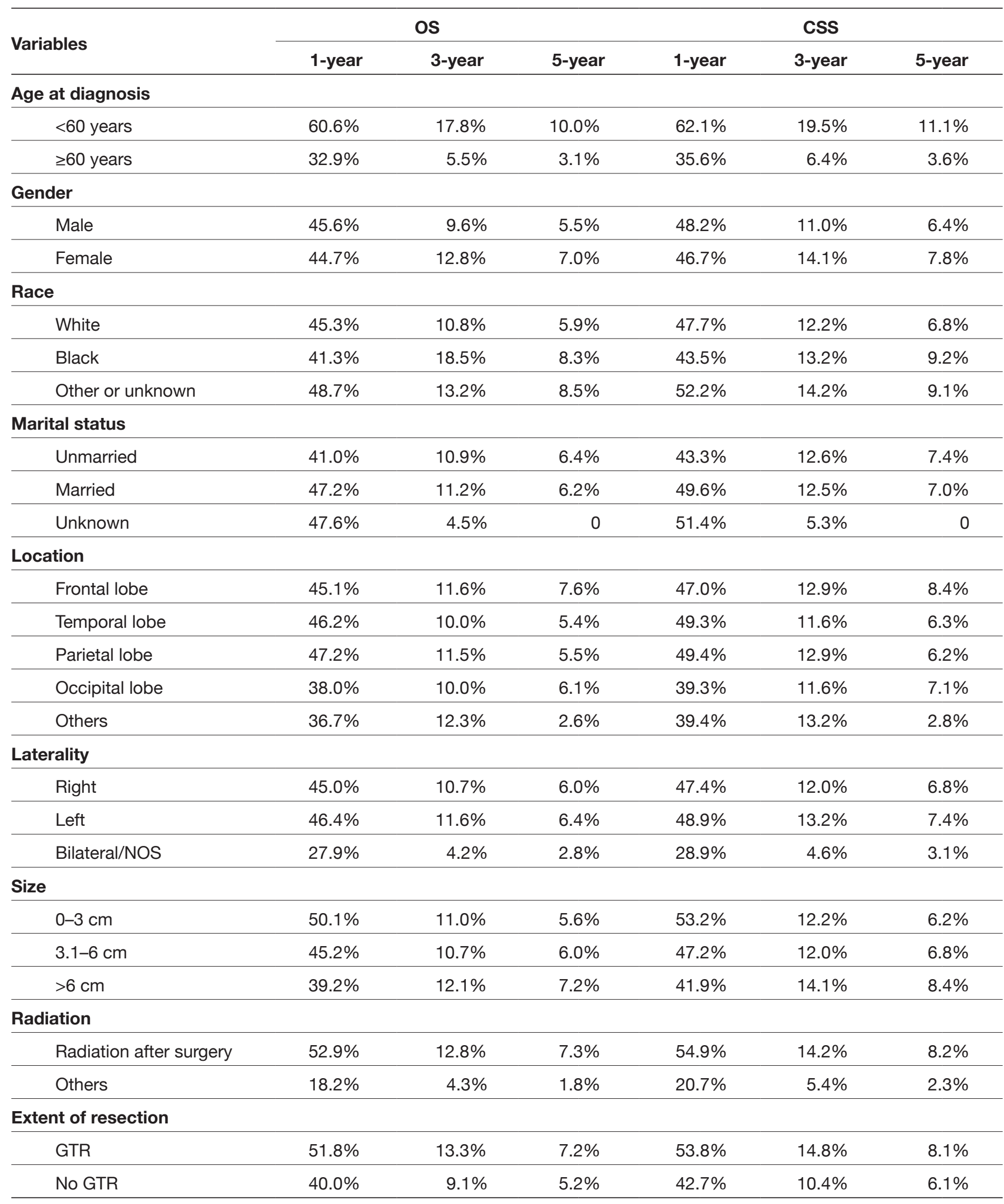

NOS: Not otherwise specified, GTR: Gross total resection. 
were shown to be positively correlated with the hazard ratios $(\mathrm{HRs})$ in the multivariate model $(\mathrm{p}<0.05)$ (Tables III and IV).

\section{Prognostic nomograms for OS and CSS}

All of the significant predictors in the Cox proportional hazards regression were incorporated to construct the nomograms of the training cohort. The prognostic nomograms for 1-, 3-, and 5-year OS and CSS are shown in Figure 4A, B. In general, both the OS and CSS rates were higher for relatively younger patients, laterality, patients with radiation after surgery, and patients with GTR treatment. Based on individual patient characteristics, it was possible to effectively predict the prognosis of patients with glioblastoma using nomograms.

\section{Validation of the nomograms}

Both internal and external validations of the nomograms were conducted using 1000 bootstrap samples. As shown in Table $\mathrm{V}$, the C-index values for the predicted OS and CSS in the internal validation cohort (training cohort) were $0.682(95 \% \mathrm{Cl}$ : $0.671-0.693)$ and $0.678(95 \% \mathrm{Cl}: 0.666-0.690)$, respectively. Whereas, the C-index values for the predicted OS and CSS in the external validation cohort (validation cohort) were

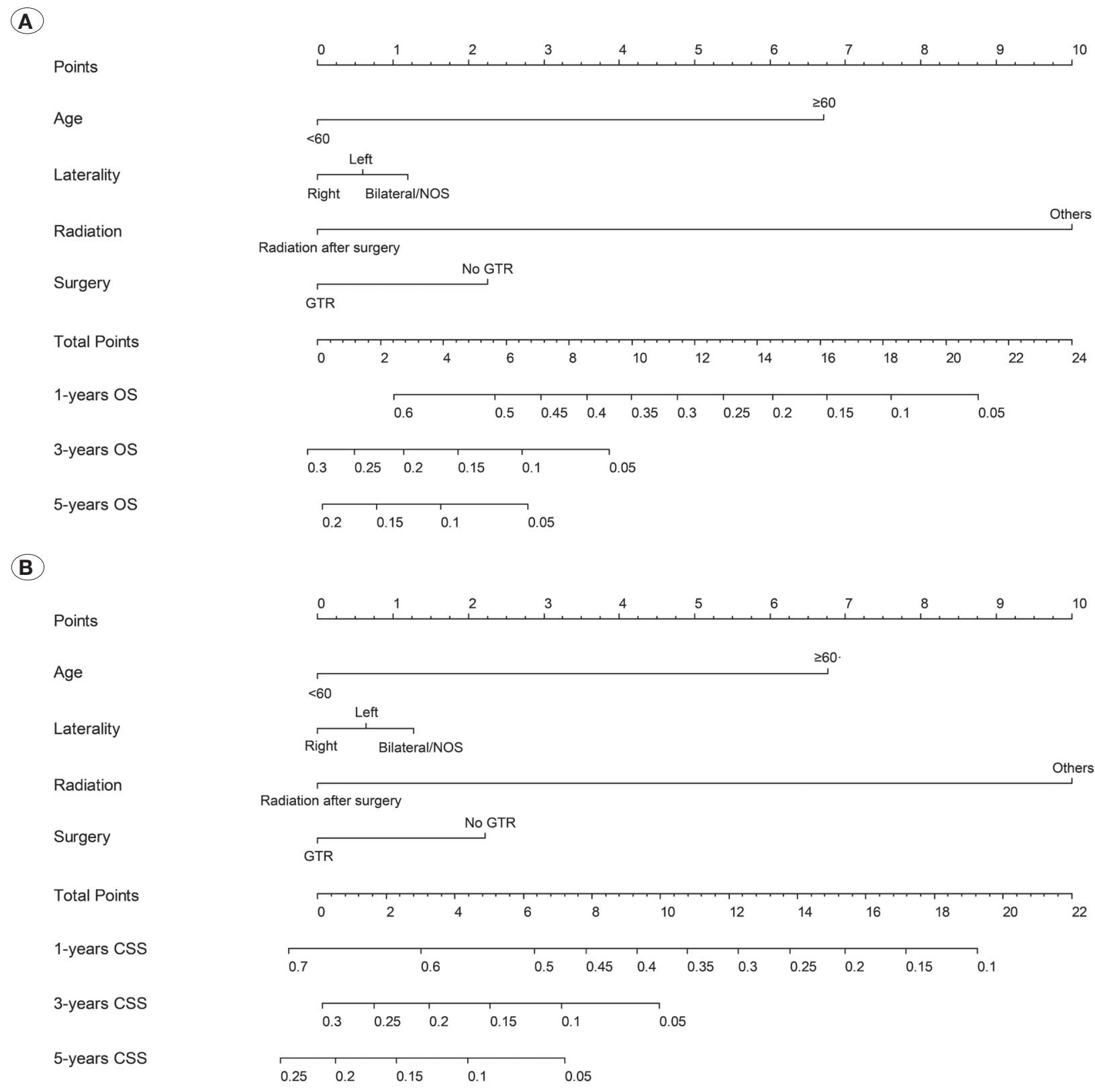

Figure 4: Nomograms for predicting the 1-, 3-, and 5-year (A) overall survival and (B) cancer-specific survival of patients with high-grade glioma. Abbreviations: OS, overall survival; CSS, cancer-specific survival. 
Xia Y. et al: Predicting Survival Rates for High-Grade Glioma

Table III: Univariate and Multivariate Analysis of Overall Survival in the Training Cohort

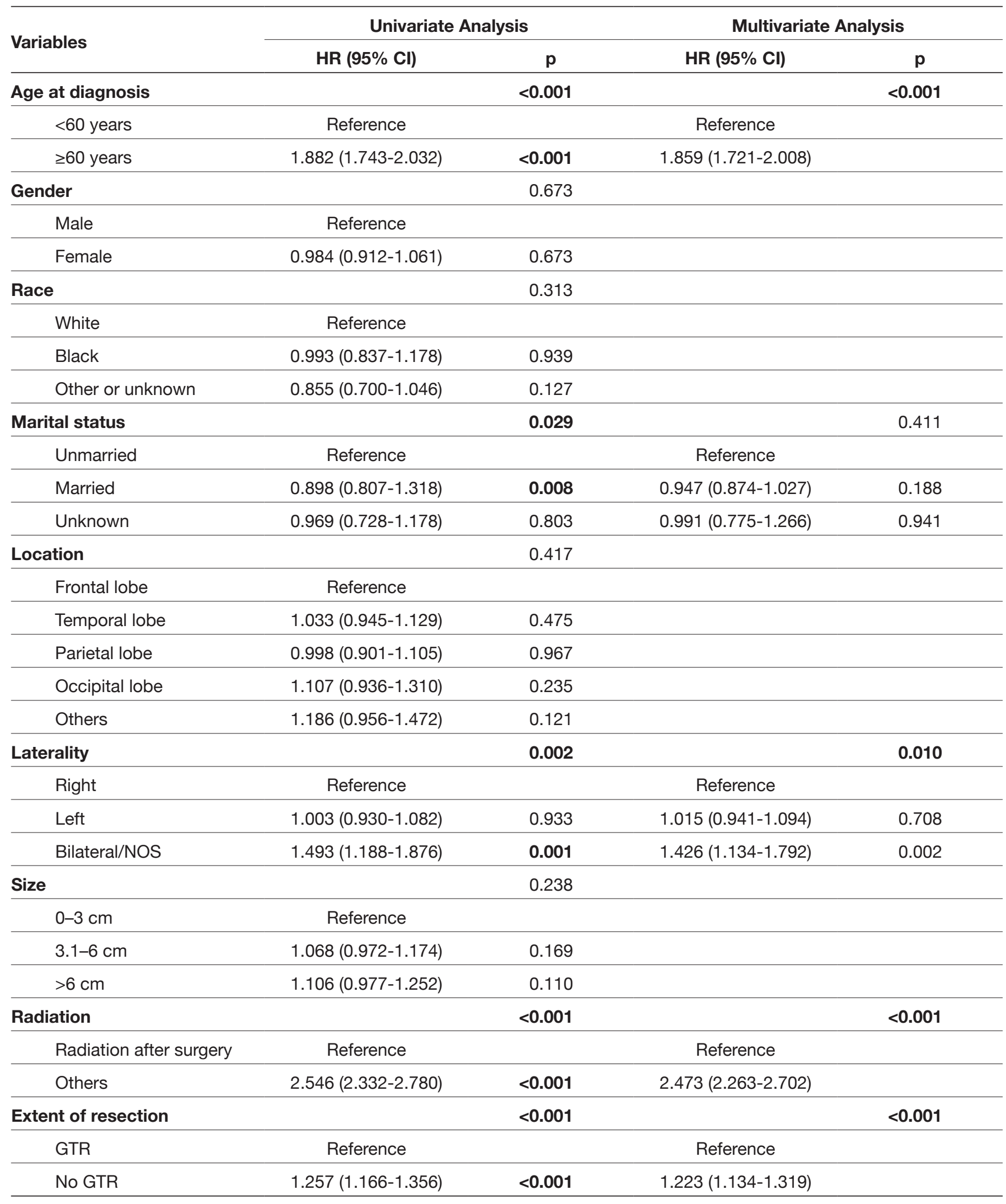

NOS: Not otherwise specified, GTR: Gross total resection. 
Table IV: Univariate and Multivariate Analysis of Cancer-Specific Survival in the Training Cohort

\begin{tabular}{|c|c|c|c|c|}
\hline \multirow{2}{*}{ Variables } & \multicolumn{2}{|c|}{ Univariate Analysis } & \multicolumn{2}{|c|}{ Multivariate Analysis } \\
\hline & HR (95\% Cl) & $\mathbf{p}$ & HR (95\% Cl) & $\mathbf{p}$ \\
\hline Age at diagnosis & & $<0.001$ & & $<0.001$ \\
\hline$<60$ years & Reference & & Reference & \\
\hline$\geq 60$ years & $1.862(1.721-2.015)$ & $<0.001$ & $1.842(1.702-1.994)$ & \\
\hline Gender & & 0.876 & & \\
\hline Male & Reference & & & \\
\hline Female & $0.994(0.919-1.074)$ & 0.876 & & \\
\hline Race & & 0.311 & & \\
\hline White & Reference & & & \\
\hline Black & $0.993(0.833-1.184)$ & 0.935 & & \\
\hline Other or unknown & $0.851(0.692-1.047)$ & 0.126 & & \\
\hline Marital status & & 0.041 & & 0.434 \\
\hline Unmarried & Reference & & Reference & \\
\hline Married & $0.899(0.828-0.977)$ & 0.012 & $0.947(0.871-1.029)$ & 0.759 \\
\hline Unknown & $0.940(0.727-1.214)$ & 0.634 & $0961(0.743-1.241)$ & 0.910 \\
\hline Location & & 0.448 & & \\
\hline Frontal lobe & Reference & & & \\
\hline Temporal lobe & $1.015(0.925-1.112)$ & 0.758 & & \\
\hline Parietal lobe & $0.993(0.894-1.103)$ & 0.895 & & \\
\hline Occipital lobe & $1.100(0.925-1.308)$ & 0.281 & & \\
\hline Others & $1.194(0.957-1.489)$ & 0.116 & & \\
\hline Laterality & & 0.001 & & 0.004 \\
\hline Right & Reference & & Reference & \\
\hline Left & $1.000(0.925-1.081)$ & 0.997 & $1.011(0.935-1.093)$ & 0.786 \\
\hline Bilateral/NOS & $1.546(1.226-1.948)$ & $<0.001$ & 1.479 (1.173-1.865) & 0.001 \\
\hline Size & & 0.270 & & \\
\hline $0-3 \mathrm{~cm}$ & Reference & & & \\
\hline $3.1-6 \mathrm{~cm}$ & $1.076(0.977-1.186)$ & 0.139 & & \\
\hline$>6 \mathrm{~cm}$ & $1.094(0.963-1.244)$ & 0.167 & & \\
\hline Radiation & & $<0.001$ & & $<0.001$ \\
\hline Radiation after surgery & Reference & & Reference & \\
\hline Others & $2.494(2.277-2.731)$ & $<0.001$ & 2.422 (2.210-2.655) & \\
\hline Extent of resection & & $<0.001$ & & $<0.001$ \\
\hline GTR & Reference & & Reference & \\
\hline No GTR & $1.247(1.154-1.347)$ & $<0.001$ & $1.213(1.122-1.311)$ & $<0.0015$ \\
\hline
\end{tabular}

NOS: Not otherwise specified, GTR: Gross total resection. 
0.685 (95\%Cl: $0.651-0.719)$ and 0.688 (95\%Cl: 0.653-0.723), respectively. These findings concluded that the nomogram models were reasonably accurate. The calibration curve plots for 1-, 3-and 5-year OS and CSS showed that the nomogram predictions did not deviate significantly from the observed outcomes in both the training and validation cohorts (Figure 5 A-F).

\section{DISCUSSION}

A proper understanding of the prognostic factors will help the individual patient to select specific treatments, and therefore it is important to the designs and interpretations of clinical trials. This study is conducted based on SEER data of adult patients with HGG diagnosed between 2000 and 2013, which is the most comprehensive training cohort up to date with clinical guiding significance for the prognosis of adult HGG. The companion prognostic factor analysis identifies several independent prognostic factors that are significantly correlated with the outcomes of postoperative radiation therapy (PRT) treatments received by patients with HGG; and, these factors include patient age at diagnosis, laterality, and the extent of resection.

It is generally accepted that patient age is one of the significant predictors of long-term survival rates of patients with HGG. In our study, there is a significant correlation between patient age at the time of diagnosis and the prognosis. Compared to younger counterparts, patients over 60 years of age have an approximately two times higher rate of both OS and CSS in first year after diagnosis, and a three times higher rate in 3 and 5 years after diagnosis. Our results are consistent with those of several previous studies. Burger et al. conducted a study to

Table V: C-Indexes for the Nomogram to Predict Overall Survival and Cancer-Specific Survival

\begin{tabular}{lcccc}
\hline \multirow{2}{*}{ Group } & \multicolumn{3}{c}{ OS } & \multicolumn{2}{c}{ CSS } \\
\cline { 2 - 5 } & C-index & $\mathbf{9 5 \% ~ C l}$ & C-index & $\mathbf{9 5 \% ~ C l}$ \\
\hline Training cohort & 0.682 & $0.671-0.693$ & 0.678 & $0.666-0.690$ \\
\hline Validation cohort & 0.685 & $0.651-0.719$ & 0.688 & $0.653-0.723$ \\
\hline
\end{tabular}
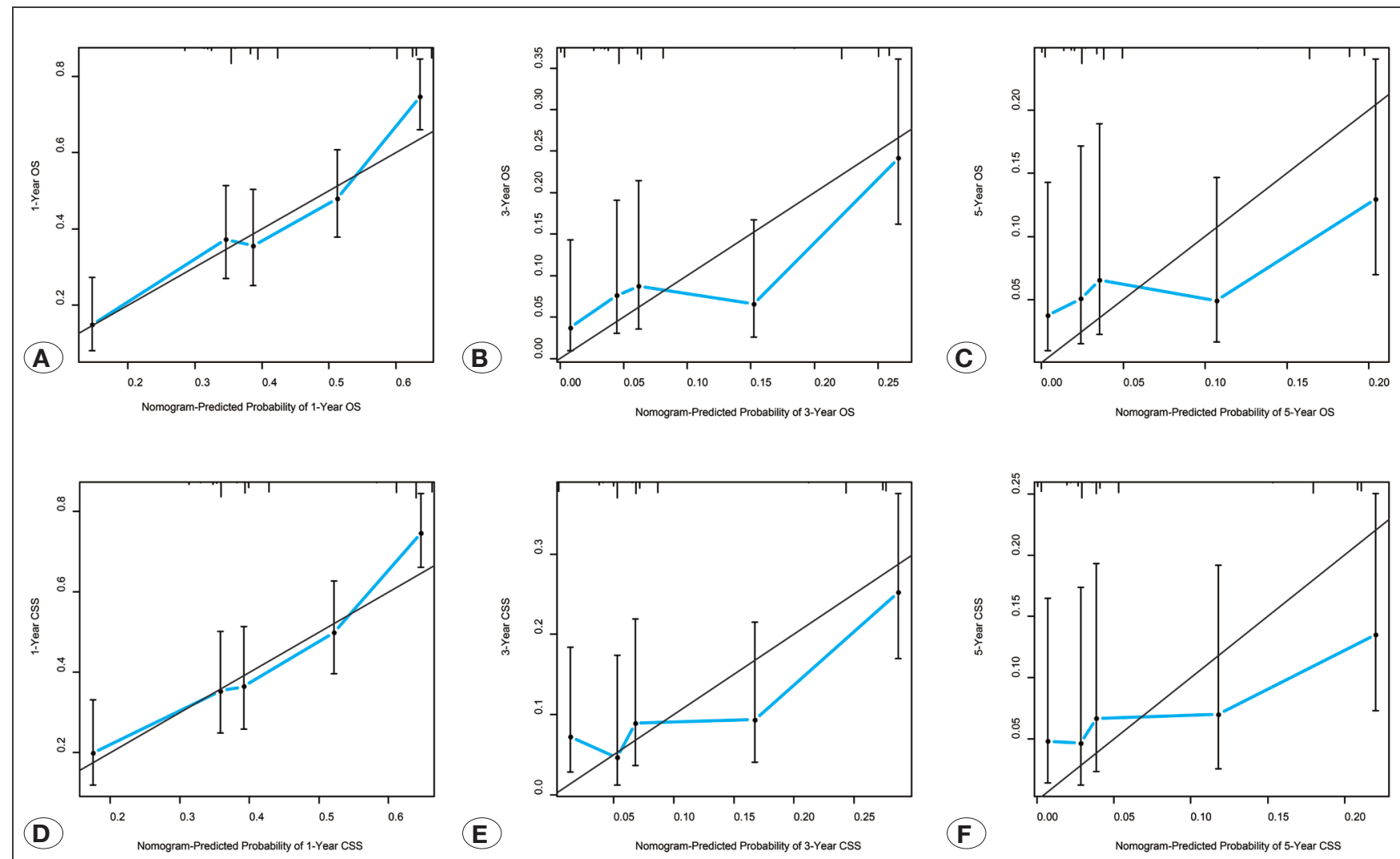

Figure 5: Internal calibration plots for A) 1-year OS, B) 3-year OS, C) 5-year OS, D) 1-year CSS, E) 3-year CSS, and F) 5-year CSS. A perfectly accurate nomogram prediction model would result in a plot where the actual and predicted probabilities for the given groups fall along the $45^{\circ}$ line. Closer distances between the pairs and the $45^{\circ}$ line indicate higher prediction accuracy. Abbreviations: OS, overall survival; CSS, cancer-specific survival. 
compare the histologic features and prognosis in glioblastoma between patients aged less than 45 years and those over 65 years. Our univariate and multivariate analysis showed the existence of a strong negative correlation between patient age at diagnosis and the length of postoperative survival $(p<0.0001)$. Moreover, morphologic change also contributes to the effect of a patient's age and the survival rate. In 2013, Walsh et al. suggested that this phenomenon is related to the ability of tumors to maintain telomeres through the canonical telomerase-based mechanism (28). In addition, it is clear that the generally poor prognosis of the elderly population is related to other factors such as inferior quality of physical status and higher rates of comorbidity, including thromboembolic events, seizures, and fluctuations in neurologic symptoms (4).

Notably, laterality is an influential factor for patients with HGG. The prognosis of patients with left or right HGGs is better than that for patients with bilateral HGGs. The mechanism of the difference between the right and left hemisphere remains unknown and needs further investigation.

The results of our study showed that PRT can significantly improve the prognosis of patients with HGG, and both the univariable and multivariable analysis indicated that patients who have PRT have significant survival advantages over nonPRT patients (HR: 2.422; 95\% Cl: 2.210-2.655; $\mathrm{p}<0.001$ ). Our study results are consistent with those of several previous studies. In the 1970s-1980s, the role of adjuvant external beam PRT for HGG treatment was first established by a series of randomized trials $(6,12,13,18,22,27,29)$. Between 1998 and 2007 , Rusthoven et al. compared survival outcomes for 14,461 patients with HGG in the SEER database whowere treated with or without adjuvant radiation therapy, and reported that adjuvant radiation therapy was associated with significant improvements in survival rates among patients with GBM by univariate and multivariate models (HR: 0.52 ; $95 \% \mathrm{Cl}$ : $0.50-$ $0.55 ; \mathrm{p}<0.001)(18)$. It is also illustrated in the American Society for Radiation Oncology Guideline (ASROG) that radiation should be initiated as soon as it is safely permissible. After surgery, PRT combined with chemotherapy should be applied to all patients (23). In addition to the fact that radiotherapy itself can improve the survival outcome of patients with HGG, the increasing accuracy of radiotherapy is also an important factor in the prognosis. Although the ionizing radiation itself has not changed, the ability to focus the beam and adapt it to the irregular contours of brain tumors, as well as minimizing the damage to nearby critical structures with intensity-modulated or image-guided techniques, have prominently improved (26).

With the advancements in surgical techniques and preoperative imaging technologies, total resection and radiotherapy have been of key importance for decades to the treatment of these lesions $(1,9,16)$. In our study, the extent of resection proves to be an independent significant predictor of the survival rate, and subtotal resection (STR) and appropriate GTR can significantly improve the prognosis for adults with HGG. Similar findings have been reported in previous studies $(6,25,29)$. In 2007, Allahdini et al. proved that both GTR and STR result in longer survival and that maximal tumor resection still remains significant in the multivariate analysis $(p<0.01)$
(1). The result of their study suggested that it is possible to maximally resect malignant gliomas within functionally critical areas without increased morbidity. In conclusion, PRT and GTR have the most significant effects on the survival outcome of patients with HGG.

The findings from our trial confirm that there is no significant difference in survival between the white and black population. Nevertheless, between 2000 and 2008, Thumma et al. studied 34,664 patients from the SEER database diagnosed with glioblastoma and found that the Asian/Pacific Islanders had a significantly better survival rate (HR: $0.83 ; 95 \% \mathrm{Cl}$ : 0.78 to $0.79 ; p \leq 0.001$ ) when compared to other populations (25). However, Barnholtz-Sloan et al. pointed out that racial difference in survival after primary glioblastoma diagnosis might be partially attributed to racial difference in treatment and income (3).

In the univariate analysis, our data showed that there exists a significant correlation between marital status and survival rate; however, this correlation did not show in the multivariate analysis. Some studies have confirmed that compared with married patients, single patients with HGG patients diagnosed with larger tumors are less likely to accept either surgical resection or postoperative radiotherapy, and thus, they would survive for a shorter period of time after diagnosis (5). Therefore, we do not consider marital status as the main factor affecting the survival rate.

The predictive function of nomograms has been used for different types of cancers, and it has even been proposed as a new standard. Our analysis is more objective and scientific than previous studies because it is able to determine the prognosis based on each patient's personal information, prognostic factors, and treatment history; but, it is also applicable for wider use. For example, a 70-year-old patient (6.8 points) who has bilateral lesions (1.2 points) and receives radiotherapy after GTR surgery (0 points) will score a total of eight points, which converts to a 1 -year OS of $15 \%$.

There are several limitations in this study as well as all other studies using the SEER database: patient treatment information, such as the modality, dose of radiotherapy, and the use of alternative therapies is inaccessible, which results in the failure to explain our results thoroughly and objectively. Therefore, these potential prognostic factors are not included in the nomogram. Moreover, comorbidities are not well documented and therefore may affect mortality among the various patient groups. Finally, similar to all national databases, there might exist miscoding of the various patients, tumors, and treatment characteristics in the SEER database (19). Despite these possible limitations, the SEER database remains a useful source of data for our research.

\section{CONCLUSION}

For patients with HGG, patient age at diagnosis, tumor location, the extent of tumor resection, and postoperative radiotherapy are the four most prominent factors that can affect the survival outcome. Further, the model we established may be a useful tool to predict the OS and the CSS of these patients. 


\section{ACKNOWLEDGMENTS}

This work is supported by grants from Natural Science Fund of Guangdong Province (No. 2017A030313597) and Southern Medical University (No. LX2016N006, No. KJ20161102).

We would like to express our sincere gratitude to Yu-Qing Tang and Ms. Sharon Arjune for providing writing assistance and giving valuable suggestions regarding this manuscript.

\section{REFERENCES}

1. Allahdini F, Amirjamshidi A, Reza-Zarei M, Abdollahi M: Evaluating the prognostic factors effective on the outcome of patients with glioblastoma multiformis: Does maximal resection of the tumor lengthen the median survival? World Neurosurg 73(2):128-134; discussion: e16, 2010

2. Balachandran VP, Gonen M, Smith JJ, DeMatteo RP: Nomograms in oncology: More than meets the eye. Lancet Oncol 16(4):e173-180, 2015

3. Barnholtz-Sloan JS, Maldonado JL, Williams VL, Curry WT, Rodkey EA, Barker FG 2nd, Sloan AE: Racial/ethnic differences in survival among elderly patients with a primary glioblastoma. J Neurooncol 85(2):171-180, 2007

4. Brandsma D, Stalpers L, Taal W, Sminia P, van den Bent MJ: Clinical features, mechanisms, and management of pseudoprogression in malignant gliomas. Lancet Oncol 9(5):453-461, 2008

5. Chang SM, Barker FG 2nd: Marital status, treatment, and survival in patients with glioblastoma multiforme: A population based study. Cancer 104(9):1975-1984, 2005

6. Fekete B, Werlenius K, Orndal C, Rydenhag B: Prognostic factors for glioblastoma patients-a clinical population-based study. Acta Neurol Scand 133(6):434-441, 2016

7. Gorlia T, van den Bent MJ, Hegi ME, Mirimanoff RO, Weller M, Cairncross JG, Eisenhauer E, Belanger K, Brandes AA, Allgeier A, Lacombe D, Stupp R: Nomograms for predicting survival of patients with newly diagnosed glioblastoma: Prognostic factor analysis of EORTC and NCIC trial 2698122981/CE.3. Lancet Oncol 9(1):29-38, 2008

8. Harrell FE Jr, Lee KL, Mark DB: Multivariable prognostic models: Issues in developing models, evaluating assumptions and adequacy, and measuring and reducing errors. Stat Med 15(4):361-387, 1996

9. Harsh GRt, Levin VA, Gutin PH, Seager M, Silver P, Wilson CB: Reoperation for recurrent glioblastoma and anaplastic astrocytoma. Neurosurgery 21(5):615-621, 1987

10. Huitzil-Melendez FD, Capanu M, O'Reilly EM, Duffy A, Gansukh B, Saltz LL, Abou-Alfa GK: Advanced hepatocellular carcinoma: Which staging systems best predict prognosis? J Clin Oncol 28(17):2889-2895, 2010

11. Kattan MW: Validating a prognostic model. Cancer 107(11):2523-2524, 2006

12. Khan L, Soliman H, Sahgal A, Perry J, Xu W, Tsao MN: External beam radiation dose escalation for high grade glioma. Cochrane Database Syst Rev 8:Cd011475, 2016
13. Kristiansen K, Hagen S, Kollevold T, Torvik A, Holme I, Nesbakken R, Hatlevoll R, Lindgren M, Brun A, Lindgren S, Notter G, Andersen AP, Elgen K: Combined modality therapy of operated astrocytomas grade III and IV. Confirmation of the value of postoperative irradiation and lack of potentiation of bleomycin on survival time: A prospective multicenter trial of the Scandinavian Glioblastoma Study Group. Cancer 47(4):649-652, 1981

14. Liang W, Zhang L, Jiang G, Wang Q, Liu L, Liu D, Wang Z, Zhu Z, Deng Q, Xiong X, Shao W, Shi X, He J: Development and validation of a nomogram for predicting survival in patients with resected non-small-cell lung cancer. J Clin Oncol 33(8):861-869, 2015

15. Louis DN, Ohgaki H, Wiestler OD, Cavenee WK, Burger PC, Jouvet A, Scheithauer BW, Kleihues P: The 2007 WHO classification of tumors of the central nervous system. Acta Neuropathol 114(2):97-109, 2007

16. Noorbakhsh A, Tang JA, Marcus LP, McCutcheon B, Gonda DD, Schallhorn CS, Talamini MA, Chang DC, Carter BS, Chen CC: Gross-total resection outcomes in an elderly population with glioblastoma: A SEER-based analysis. J Neurosurg 120(1):31-39, 2014

17. Ostrom QT, Gittleman H, Fulop J, Liu M, Blanda R, Kromer C, Wolinsky Y, Kruchko C, Barnholtz-Sloan JS: CBTRUS statistical report: Primary brain and central nervous system tumors diagnosed in the United States in 2008-2012. Neuro Oncol 17 Suppl 4:iv1-iv62, 2015

18. Rusthoven CG, Carlson JA, Waxweiler TV, Dally MJ, Baron AE, Yeh N, Gaspar LE, Liu AK, Ney DE, Damek DM, Lillehei $\mathrm{KO}$, Kavanagh BD: The impact of adjuvant radiation therapy for high-grade gliomas by histology in the United States population. Int J Radiat Oncol Biol Phys 90(4):894-902, 2014

19. Sampson JH, Lad SP, Herndon JE 2nd, Starke RM, Kondziolka D: SEER insights. J Neurosurg 120(2):297-298, 2014

20. Shi X, Hu WP, Ji QH: Development of comprehensive nomograms for evaluating overall and cancer-specific survival of laryngeal squamous cell carcinoma patients treated with neck dissection. Oncotarget 8(18):29722-29740, 2017

21. Stewart LA: Chemotherapy in adult high-grade glioma: $A$ systematic review and meta-analysis of individual patient data from 12 randomised trials. Lancet 359(9311):1011-1018, 2002

22. Stupp R, Brada M, van den Bent MJ, Tonn JC, Pentheroudakis G: High-grade glioma: ESMO clinical practice guidelines for diagnosis, treatment and follow-up. Ann Oncol 25 Suppl 3:iii93-101, 2014

23. Sulman EP, Ismaila N, Chang SM: Radiation therapy for glioblastoma: American Society of Clinical Oncology Clinical Practice Guideline Endorsement of the American Society for Radiation Oncology Guideline. J Oncol Pract 13(2):123-127, 2017

24. Tang LQ, Li CF, Li J, Chen WH, Chen QY, Yuan LX, Lai XP, He Y, Xu YX, Hu DP, Wen SH, Peng YT, Zhang L, Guo SS, Liu LT, Guo L, Wu YS, Luo DH, Huang PY, Mo HY, Xiang YQ, Sun R, Chen MY, Hua YJ, Lv X, Wang L, Zhao C, Cao KJ, Qian CN, Guo $X$, Zeng $Y X$, Mai $H Q$, Zeng MS: Establishment and validation of prognostic nomograms for endemic nasopharyngeal carcinoma. J Natl Cancer Inst 108(1): pii: djv291, 2016 
25. Thumma SR, Fairbanks RK, Lamoreaux WT, Mackay AR, Demakas JJ, Cooke BS, Elaimy AL, Hanson PW, Lee CM: Effect of pretreatment clinical factors on overall survival in glioblastoma multiforme: A Surveillance Epidemiology and End Results (SEER) population analysis. World J Surg Oncol 10:75, 2012

26. Van Meir EG, Hadjipanayis CG, Norden AD, Shu HK, Wen $\mathrm{PY}$, Olson JJ: Exciting new advances in neuro-oncology: The avenue to a cure for malignant glioma. CA Cancer J Clin 60(3):166-193, 2010

27. Walker MD, Alexander E Jr, Hunt WE, MacCarty CS, Mahaley MS Jr, Mealey J Jr, Norrell HA, Owens G, Ransohoff J, Wilson CB, Gehan EA, Strike TA: Evaluation of BCNU and/ or radiotherapy in the treatment of anaplastic gliomas. A cooperative clinical trial. J Neurosurg 49(3):333-343, 1978
28. Walsh KM, Rice T, Decker PA, Kosel ML, Kollmeyer T, Hansen HM, Zheng S, McCoy LS, Bracci PM, Anderson E, Hsuang G, Wiemels JL, Pico AR, Smirnov I, Molinaro AM, Tihan T, Berger MS, Chang SM, Prados MD, Lachance DH, Sicotte H, EckelPassow JE, Wiencke JK, Jenkins RB, Wrensch MR: Genetic variants in telomerase-related genes are associated with an older age at diagnosis in glioma patients: Evidence for distinct pathways of gliomagenesis. Neuro Oncol 15(8):1041-1047, 2013

29. Yang W, Xu T, Garzon-Muvdi T, Jiang C, Huang J, Chaichana KL: Survival of ventricular and periventricular high-grade gliomas: a surveillance, epidemiology, and end results program-based study. World Neurosurg 111:e323-e334, 2018

30. Zhang $\mathrm{Y}$, Zhang J, Zeng H, Zhou XH, Zhou HB: Nomograms for predicting the overall and cancer-specific survival of patients with classical Hodgkin lymphoma: A SEER-based study. Oncotarget 8(54):92978-92988, 2017 